\title{
TERMINAÇÃO DE BOVINOS BRANGUS INTEIROS E CASTRADOS COM CANA-DE- AÇÚCAR (SACCHARUM OFFICINARUM) OU SILAGEM DE CAPIM MOMBAÇA (PANICUM MAXIMUM CV MOMBAÇA)
}

Marcelo Diniz dos Santos ${ }^{1}$; Fabiola Cristine de Almeida Rego ${ }^{2}$, Marcos Vinicius de Lima Rodrigues ${ }^{1}$, Deiler Sampaio Costa ${ }^{3}$, Caue Natam de Souza ${ }^{1}$, Luiz Fernando Coelho cunha Filho ${ }^{2}$, Laís Belan ${ }^{2}$, Marilice Zundt ${ }^{4}$, Jorge Luiz Santana ${ }^{5}$

${ }^{1}$ UNIC, Faculdade de Medicina Veterinária - UNIC, Cuiabá-MT E-mail: dinizms@uol.com.br; viniciusvado@hotmail.com.22UNOPAR, Arapongas, PR. E-mail: fabíola_rego@yahoo.com.br; luiz.cunha@unopar.br. ${ }^{3}$ Faculdade de Medicina Veterinária e Zootecnia - UFMS E-mail: deilercosta@yahoo.com.br. ${ }^{4}$ Universidade do Oeste Paulista - UNOESTE - Presidente Prudente -SP, E-mail - mari@unoeste.br. ${ }^{5}$ Medico Veterinário, Fazenda GAP Sereno, Jaciara-MT. E-mail sereno@vsp.com.br

\section{RESUMO}

O objetivo deste trabalho foi avaliar o consumo, o ganho de peso, a conversão alimentar e a viabilidade econômica da utilização de cana de açúcar in natura e da silagem de capim mombaça na terminação de novilhos Brangus inteiros e castrados. Utilizaram-se 140 bovinos Brangus, com média de 21 meses e $355 \mathrm{~kg}$ de peso vivo, identificados e distribuídos aleatoriamente nos tratamentos: T1, 37 animais inteiros e T2, 37 animais castrados, ambos recebendo cana-de-açúcar (Saccharum officinarum) triturada in natura; T3, 33 animais inteiros e T4, 33 animais castrados, ambos recebendo silagem de capim mombaça (Panicum maximum cv mombaça).Para avaliação econômica, levantaram-se os custos completos para a produção dos volumosos oriundos da própria fazenda, foram consideradas também despesas com alimentos concentrados, diesel, mãode-obra, manutenção de equipamentos e controle de parasitas. O peso final dos animais inteiros foi maior $(P<0,05)$ que dos animais castrados, e a conversão alimentar foi maior nos animais castrados, o que indica que os animais inteiros foram mais eficientes na transformação do alimento em ganho de peso. A maior receita líquida observada nos animais inteiros (mombaça) se justifica pelo maior ganho de peso e menor custo das dietas. Os animais da dieta contendo silagem de capim mombaça apresentaram maior viabilidade econômica do que os submetidos a dieta com cana-de-açúcar.

Palavras-chave: bovinos de corte; confinamento; ganho de peso; conversão alimentar; viabilidade econômica.

\section{FINISHING OF CATTLE BRANGUS INTACT MALE AND CASTRATED WITH SUGAR CANE (SACCHARUM OFFICINARUM) OR MOMBAÇA GRASS SILAGE (PANICUM MAXIMUM CV MOMBAÇA)}

\begin{abstract}
The aim of this study was to evaluate the intake, weight gain, feed:gain ratio, carcass quality and economic viability of using fresh sugar cane and mombaça grass silage in Brangus castrated and intact males on termination. We used 140 Brangus cattle, with an average of 21 months and 355 kg live weight, identified and randomly assigned to treatments: T1, 37 intac males and T2, 37 castrated animals receiving both fresh sugar cane (Saccharum officinarum ) crushed;T3, 33 intact males and T4, 33 castrated animals, both fed mombaça grass silage (Panicum maximum cV mombaça). For economic evaluation, it was estimated the production costs of bulky coming from the farm costs, expenses concentrates, diesel, hand labor, equipment maintenance and control of
\end{abstract}


parasites were also considered. The final weight of the entire animal was higher $(P<0.05)$ than castrated animals. And feed conversion was higher in castrated animals, indicating that the bulls were more efficient in converting food into weight gain. The higher net revenue observed in intact males (mombaça) is justified by the greater weight gain and lower diet costs. Animals that received diet with mombaça grass silage had greater economic feasibility of that received diet with sugar cane.

Keywords: cattle; confinement; weight gain; feed:gain ratio; economic viability. 


\section{INTRODUÇÃO}

A bovinocultura de corte se destaca no Brasil como a principal fonte de proteína de origem animal para a população e, apesar da sua produtividade vêm buscando melhores índices com o uso de manejos alimentares mais intensivos, em decorrência da situação de degradação dos pastos, que ocorre em grande parte do país. Neste sentido o confinamento e o semiconfinamento vêm se destacando como opções de terminação de bovinos, pois reduzem o ciclo pecuário e proporcionam rapidez no giro de capital do pecuarista.

Atualmente a competitividade do mercado de carne e as exigências dos consumidores por produtos com menor teor de gordura, tem forçado os pecuaristas a desenvolverem e adotarem tecnologias visando aumentar a produtividade e produção de carne, reduzir o tempo de abate dos animais e principalmente produzir animais com menor cobertura de gordura. Porém, adotar um manejo intensivo objetivando maior produtividade deve estar aliado a outros fatores, como o potencial genético dos animais e estratégias de alimentação que atendam às exigências nutricionais (BURGUI, 2001).

Com relação aos volumosos disponíveis no Brasil, a cana-de-açúcar (Saccharum officinarum) se destaca por ser uma gramínea perene com alta produção de matéria verde (80 a 180 ton/ha/ano), constituindo-se em importante fonte energética de baixo custo (NDT entre 56 a $62 \%$ ). Contudo, possui baixo teor de PB (2 a $4 \%$ na MS) e de minerais, principalmente de fósforo (0,06 a 0,08\%) (FARIA et al., 1993). Entretanto, quando corretamente associada com concentrados, a cana-de-açúcar pode ser uma alternativa para bovinos em confinamento, mesmo tendo baixo valor nutricional (FERNANDES et al., 2007).

Outra opção de volumoso em crescente utilização é a produção de silagens de capins tropicais de elevada produtividade, apresentando vantagens em relação a outros volumosos, como algumas pesquisas vêm demonstrando com o uso de capim tanzânia e capim elefante (SANTOS, ZANINE, 2006; ZANINE et al., 2007).

Aliado a produtividade dos animais, outro foco das pesquisas, é a qualidade da carne ofertada aos consumidores, que pode ser influenciada pelo sexo do animal, idade, tamanho corporal e o sistema de produção. A influencia mais pronunciada do sexo sobre a composição da carcaça é alcançada por meio do processo de engorda. Sendo assim, diversos estudos indicam que animais inteiros têm maior taxa de crescimento, melhor conversão alimentar e produzem carcaça com menor cobertura de gordura (RESTLE et al., 2000; SILVA, 2000). 
Considerando a disponibilidade destes volumosos no sistema produtivo, a necessidade de estudos regionais sobre 0 referido assunto e os dados de avaliação econômica, este trabalho tem como objetivo avaliar o consumo, o ganho de peso, a conversão alimentar e a viabilidade econômica da utilização de cana de açúcar in natura e da silagem de capim mombaça na terminação de novilhos Brangus inteiros e castrados.

\section{MATERIAL E MÉTODOS}

O experimento foi realizado na fazenda Sereno (Jaciara-MT) nos meses de julho a outubro. Utilizaram-se 140 bovinos Brangus, com média de 21 meses e $355 \mathrm{~kg}$ de peso vivo, identificados e distribuídos aleatoriamente nos tratamentos: T1, 37 animais inteiros e T2, 37 animais castrados, ambos recebendo cana-de-açúcar (Saccharum officinarum) triturada In natura; T3, 33 animais inteiros e T4, 33 animais castrados, ambos recebendo silagem de capim mombaça (Panicum maximum cv mombaça). Os animais de cada tratamento foram mantidos em áreas de $2500 \mathrm{~m}^{2}$, com bebedouro (água ad libitum) e cocho para volumoso. O teor de matéria seca (MS) da cana-de-açúcar in natura foi de $23,65 \%$ e o da silagem de mombaça $28,4 \%$.

As dietas foram formuladas para ganhos estimados de 1200 g/animal/dia (NATIONAL RESEARCH COUNCIL-NRC, 2000) sendo isoproteícas e isoenergéticas, reformuladas a cada 30 dias (Período experimental) para atender às exigências dos animais conforme o ganho de peso obtido.

O concentrado utilizado em ambas as dietas foi composto por milho, farelo de soja, caroço de algodão, calcita, sal mineral e ionóforo, e sua proporção variou de 55 a 62\% (dieta com cana) e 52 a 60\% (dieta com silagem de mombaça) durante o experimento (Tabela 1). 
Tabela 1. Composição percentual dos ingredientes (\% MS) utilizados nas dietas, por período experimental, contendo cana-de-açúcar e silagem de capim mombaça

\begin{tabular}{lcccccc}
\hline & \multicolumn{3}{c}{ Cana de açúcar } & \multicolumn{3}{c}{ Silagem mombaça } \\
\hline Ingredientes (\%) & 30 dias & 60 dias & 90 dias & 30 dias & 60 dias & 90 dias \\
\hline Cana-de-açúcar & 45,00 & 40,00 & 37,66 & -------- & ------- & -------- \\
Silagem mombaça & -------- & -------- & ------- & 48,00 & 42,28 & 39,93 \\
Fubá de milho & 41,27 & 39,00 & 42,00 & 40,00 & 37,92 & 42,00 \\
Farelo de Soja & 10,96 & 5,11 & 4,77 & 4,72 & & \\
Caroço de algodão & 1,27 & 14,13 & 14,07 & 6,28 & 17,87 & 17,07 \\
Sal mineral $^{*}$ & 1,00 & 1,00 & 1,00 & 1,00 & 1,00 & 1,00 \\
Calcita $^{* *}$ & 0,50 & 0,76 & 0,50 & ------- & 0,93 & -------- \\
lonóforo $^{*}$ & 0,03 & 0,03 & 0,03 & 0,03 & 0,03 & 0,03 \\
\hline
\end{tabular}

"Minerais contidos em cada kg: 150 g Ca, 85 g P, 127 g Na, 5 g Mg, 23 g S, 500 mg Mn, 5500 mg Zn, 1661 mg Cu, 200 mg Co, $171 \mathrm{mg} \mathrm{l}, 27 \mathrm{mg}$ Se.

${ }^{* *}$ Percentagem de ionóforo na mistura do concentrado

Amostras dos alimentos foram retiradas e enviadas ao Laboratório de Nutrição da Universidade Federal do Paraná e apresentaram níveis de proteína bruta (PB) de 10,5 e $11,0 \%$ (início e final do experimento, respectivamente) e as estimativas de nutrientes digestíveis totais (NDT) foram de 71 e 73\% (início e final do experimento, respectivamente). O NDT foi estimado segundo Capelle et al. (2001).

As dietas foram fornecidas quatro vezes ao dia, às 7:00, 10:00, 15:00 e 18:00 h sendo as sobras pesadas antes do primeiro trato. Para obtenção do consumo alimentar utilizaram-se dois vagões forrageiros com peso pré-definido dos volumosos e respectivos níveis ocupados, onde foram adicionados os concentrados, previamente pesados, obtendo-se o peso diário dos alimentos fornecidos aos animais.
Os animais tiveram 15 dias de adaptação às dietas e após iniciou-se a fase experimental com duração de 89 dias (28/07 a $25 / 10)$. Os animais foram pesados no início e final do experimento e a cada 30 dias (período experimental), sempre antes da primeira refeição, para avaliar o ganho de peso.

Para avaliação econômica, levantouse os custos completos para a produção dos volumosos oriundos da própria fazenda, sendo todos convertidos em dólar, na ocasião cotado em U\$2,31. A silagem de capim mombaça utilizada no experimento foi produzida no sistema integração (lavourapecuária), sendo o custo obtido de $U \$ 30,30$ por tonelada, considerando plantio, manutenção (9 anos), colheita e ensilagem, com produção de 60 ton/ha/corte ano (MN). 
Para a cana considerou-se plantio, manutenção por quatro anos e cortes, com produção de 80 ton/ha/corte (MN) e custo de U\$ 20,34 por tonelada. Os demais constituintes da dieta foram adquiridos pelos seguintes preços por tonelada: milho (U\$ $160,00)$, farelo de soja (U\$ 394,00), caroço de algodão (U\$ 151,50), sal mineral (U\$ 662,30), calcita (R\$ 13,00) e ionóforo (U\$ 23.918,00).As despesas com diesel (corte e distribuição da cana e da silagem), mão-deobra (três funcionários), manutenção de equipamentos e controle de endo e ectoparasitos foram divididas por 500 animais existentes em todo o confinamento, para se obter o custo parcial dos bovinos deste estudo. A depreciação das instalações não foi considerada em função do tempo de construção e de utilização no experimento.

Adotou-se o percentual médio de 53\% de rendimento de carcaça dos animais abatidos conforme dados do frigorífico.

As análises estatísticas das variáveis foram interpretadas por análise de variância e as médias comparadas pelo teste de Tukey, a $5 \%$ de probabilidade, e a viabilidade econômica foi avaliada por análise descritiva.

\section{RESULTADOS E DISCUSSÃO}

Não houve diferença $(P>0,05)$ nos pesos iniciais e no consumo médio de matéria seca dos bovinos alimentados com cana-de-açúcar e capim mombaça, tanto para os animais inteiros e castrados (Tabela 2), e os valores estão próximos aos estimados pelo National Research Council-NRC (2000). O peso final (Tabela 2) dos animais inteiros foi maior $(P<0,05)$ que dos animais castrados nas duas dietas e também apresentaram maior $(P<0,05)$ ganho de peso diário em relação aos castrados, sendo 14 e $16 \%$ mais produtivos que os castrados nas dietas contendo cana e silagem de mombaça, respectivamente.

Esse mesmo padrão de resposta das variáveis foi obtido por Restle et al. (2000), que ao avaliarem animais inteiros ou castrados dos grupos genéticos Charolês e Nelore, observaram que não houve diferença entre inteiros e castrados com relação ao consumo voluntário de matéria seca, entretanto os animais inteiros tiveram ganhos superiores em $13,7 \%$ com relação aos castrados. E a conversão alimentar foi maior nos animais castrados, o que indica que os animais inteiros foram mais eficientes na transformação do alimento em ganho de peso.

Como possível reflexo das diferenças no ganho de peso diário, verificou-seque os animais inteiros tiveram melhor $(P<0,05)$ conversão alimentar (6,5 com cana e 6,1 com mombaça) que os castrados (7,6 com cana e 7,3 com mombaça), resultando em maior eficiência alimentar dos animais inteiros 
alimentados com cana $(15,3 \%)$ e mombaça (16,\%), em relação aos castrados.

Os animais inteiros consumiram $16,9 \%$ e $19,6 \%$ menos alimento que os castrados para adicionar $1 \mathrm{~kg}$ no peso vivo, nas dietas com cana de açúcar e silagem de capim mombaça, respectivamente. Demonstrando assim que o uso de animais inteiros, independente da dieta, traz vantagens não apenas no ganho de peso dos animais, mas na eficiência alimentar; o que pode garantir a viabilidade de um sistema de confinamento, em que grande parte dos custos é representada pela dieta. Da mesma forma Fernandes et al. (2007) testando dietas a base de cana de açúcar para bovinos jovens da raça Canchim, também verificaram maior eficiência para os animais inteiros. Essa melhor eficiência, demonstrada por animais inteiros, é devido a produção de hormônios androgênicos, como a testosterona, produzida nos testículos (Field, 1971); o que para a produção de carne, segundo Restle et al. (2000), uma alternativa viável para tornar o sistema de produção mais eficiente.

Os resultados de conversão alimentar obtidos nesse estudo, são semelhantes aos de Gesualdi Júnior et al. (2000), avaliando novilhos inteiros Limousin $x$ Nelore, que observaram em dietas com 62,5 e $75 \%$ de concentrado conversões alimentares de 6,39 e 6,67, respectivamente.

TABELA 2. Médias do peso inicial e final, ganho de peso diário (GPD, kg), consumo de matéria seca (CMS, kg MS/dia), consumo de matéria seca em relação ao peso vivo (CPV,\%) e conversão alimentar (CA) expressa em kg de MS consumida/kg de peso vivo dos bovinos inteiros e castrados submetidos às dietas com cana-de-açúcar e silagem de capim mombaça, durante 89 dias de confinamento

\begin{tabular}{lccccc}
\hline Discriminação & \multicolumn{3}{c}{ Cana-de-açúcar } & & \multicolumn{2}{c}{ Silagem mombaça } & \\
\cline { 2 - 3 } \cline { 5 - 6 } & Inteiros & Castrados & & Inteiros & Castrados \\
\hline Peso inicial (kg) & $363 \pm 34,1 \mathrm{~A}$ & $349 \pm 25,4 \mathrm{~A}$ & & $360 \pm 29,7 \mathrm{~A}$ & $346 \pm 28,2 \mathrm{~A}$ \\
Peso final (kg) & $483 \pm 46,1 \mathrm{~A}$ & $452 \pm 29,9 \mathrm{~B}$ & & $487 \pm 38,8 \mathrm{~A}$ & $453 \pm 37,1 \mathrm{~B}$ \\
GPD (kg) & $1,35 \pm 0,21 \mathrm{~A}$ & $1,16 \pm 0,17 \mathrm{~B}$ & & $1,43 \pm 0,21 \mathrm{~A}$ & $1,20 \pm 0,24 \mathrm{~B}$ \\
CMS(Kg MS/dia) & $8,82 \pm 0,19 \mathrm{~A}$ & $8,82 \pm 0,18 \mathrm{~A}$ & & $8,78 \pm 0,20 \mathrm{~A}$ & $8,77 \pm 0,18 \mathrm{~A}$ \\
CPV (\% PV) & $2,1 \pm 0,07 \mathrm{~A}$ & $2,2 \pm 0,09 \mathrm{~B}$ & & $2,1 \pm 0,06 \mathrm{~A}$ & $2,2 \pm 0,08 \mathrm{~B}$ \\
CA & $6,5 \pm 0,58 \mathrm{C}$ & $7,6 \pm 0,74 \mathrm{~A}$ & & $6,1 \pm 0,61 \mathrm{D}$ & $7,3 \pm 0,68 \mathrm{~B}$ \\
\hline
\end{tabular}

Médias seguidas por letras diferentes nas linhas diferem entre si a $5 \%$ pelo teste de Tukey.

A avaliação econômica (Tabela 3) demonstrou maior viabilidade para o uso de animais inteiros em relação aos castrados no confinamento, seja na dieta com cana de açúcar ou com a silagem de capim mombaça, apresentando diferença de receita liquida (DRL) de U\$ 25,98 e 30,30para tais dietas, respectivamente. 
O custo total com alimentação em todo o período de confinamento variou entre diferentes tipos de dietas, sendo U\$121,46 para a cana e U\$110,17 para a silagem de mombaça correspondendo a 84,4 e $83,3 \%$ dos custos totais do confinamento, respectivamente. Os outros custos do confinamento foram iguais nos dois tratamentos.

No confinamento com cana de açúcar a receita líquida (Tabela 3 ) dos animais inteiros foi $68 \%$ superior aos castrados; enquanto no grupo confinado com silagem de mombaça, a superioridade da receita dos inteiros em relação aos castrados foi de $48 \%$. Isto ocorreu em função dos bovinos inteiros terem obtido maior $(P<0,05)$ ganho de peso sem alterar os custos de produção.

Comparando o quatro grupos (inteiros e castrados nas duas dietas), a maior receita líquida (Tabela 3) foi obtida nos animais inteiros confinados com silagem de mombaça, o que pode ser atribuído a menor conversão alimentar deste grupo (Tabela 2), aliado também ao custo inferior com alimentação.

TABELA 3. Avaliação econômica da terminação de bovinos castrados (C) e inteiros (I) submetidos a dietas com cana-de-açúcar e silagem de capim mombaça, durante 89 dias de confinamento

\begin{tabular}{|c|c|c|c|c|}
\hline \multirow{2}{*}{$\begin{array}{l}\text { Discriminação } \\
\text { Variáveis de Custos }^{1}\end{array}$} & \multicolumn{2}{|c|}{ Cana-de-açúcar } & \multicolumn{2}{|c|}{ Silagem mombaça } \\
\hline & $\mathrm{I}$ & $\mathrm{C}$ & 1 & $\mathrm{C}$ \\
\hline Diesel (U\$/cab/ 89 dias) & 7,59 & 7,59 & 7,59 & 7,59 \\
\hline Mão-de-obra (U\$/ cab/ 89 dias) & 8,44 & 8,44 & 8,44 & 8,44 \\
\hline Manutenção equipamentos (U\$/cab/89 dias) & 2,12 & 2,12 & 2,12 & 2,12 \\
\hline Controle de parasitos (U\$/cab/89 dias) & 1,40 & 1,40 & 1,40 & 1,40 \\
\hline Alimentação (U\$/cab/89 dias) & 121,46 & 121,46 & 110,17 & 110,17 \\
\hline Eventuais (U\$/cab/89 dias) & 2,81 & 2,81 & 2,81 & 2,81 \\
\hline Despesas totais (U\$/cab/89 dias ) & 143,82 & 143,82 & 132,53 & 132,53 \\
\hline \multicolumn{5}{|l|}{ Variáveis de Receita $^{2}$} \\
\hline Ganho de peso diário (kg) & $1,35^{\mathrm{A}}$ & $1,16^{\mathrm{B}}$ & $1,43^{\mathrm{A}}$ & $1,20^{B}$ \\
\hline Ganho peso 89 dias (kg) & 120 & 103 & 127 & 107 \\
\hline Ganho total @ (53\% rendimento) & 4,2 & 3,6 & 4,5 & 3,8 \\
\hline Receita total (U\$/ cab) ${ }^{*}$ & 181,81 & 155,84 & 194,80 & 164,50 \\
\hline Receita liquida (U\$/ cab) & 37,98 & 12,00 & 62,51 & 32,21 \\
\hline DRL (U\$/ cab) & \multicolumn{2}{|c|}{25,98} & \multicolumn{2}{|c|}{30,30} \\
\hline
\end{tabular}

${ }^{1}$ Média do valor dólar no período experimental $\mathrm{R} \$ 2,31$

${ }^{2}$ Preço por @ = U\$ 43,29; DRL - Diferença de receita líquida entre animais inteiros e castrados nas dietas com cana-deaçúcar e silagem capim mombaça

${ }^{A, B}$ Médias seguidas por letras diferentes na linha diferem entre si a 5\% pelo teste de Tukey.

Considerando as observações visuais ocorridas durante o período experimental em que ocorreu aumento da matéria seca (folhas secas) e redução na quantidade de folhas da 
cana de açúcar (em função do período seco), que possivelmente acarretou variações na sua digestibilidade. Fatos estes já relatados por Farias et al. (1995) como fatores que podem interferir no consumo e no ganho de peso dos animais. Estes fatores não ocorrem com produtos ensilados, ficando a silagem de mombaça com padrão de qualidade semelhante ao longo de todo o período de confinamento dos animais. Além disso, em virtude do menor valor nutricional da cana em relação à da silagem de mombaça foi necessário maior investimento em concentrado, por isto o custo da alimentação tornou-se maior, proporcionando menor rentabilidade econômica da dieta com cana em relação a silagem de mombaça.

Segundo Missio et al. (2009), o lucro do confinamento para terminação de bovinos é variável, visto que o mercado de insumos e o preço do produto final sofrem muitas variações. Estes autores, ao avaliarem a viabilidade econômica do uso de diferentes níveis de concentrado na dieta no confinamento de tourinhos para abate, verificaram que os custos de produção aumentaram com a participação do concentrado na dieta, em resposta à elevação dos custos com concentrado. Dados que corroboram com o presente estudo, quando se explica o elevado custo de alimentação para os animais tratados com cana-de-açúcar, devido ao aumento na quantidade de concentrado para balancear a dieta.

A superioridade dos animais inteiros em relação aos castrados também tem sido relatadas em animais terminados a pasto. Ítavo et al. (2008) realizaram a avaliação econômica de bovinos terminados em pastagens de Brachiaria decumbens, e verificaram que a castração influenciou negativamente na rentabilidade do sistema, sendo as respostas mais favoráveis ao grupo dos animais inteiros, com maior quantidade de carcaça por animal e por hectare em relação aos não-castrados. Confirmou-se ainda neste trabalho, maior lucratividade para este mesmo grupo.

Considerando as vantagens produtivas da terminação de bovinos inteiros e a escassez de dados de viabilidade econômica com diferentes fontes de volumosos, outros estudos podem ser realizados com $\mathrm{o}$ intuito de fornecer gradiente de respostas que auxiliem na tomada de decisão quanto ao sistema de terminação a ser utilizado.

\section{CONCLUSÕES}

Bovinos inteiros obtiveram maior ganho de peso, melhor conversão alimentar e maior rentabilidade econômica que os castrados.Os animais da dieta contendo silagem de capim mombaça apresentaram 
maior viabilidade econômica do que os submetidos a dieta com cana-de-açúcar.

\section{REFERÊNCIAS}

BURGÜI, R. Confinamento estratégico. In: MATTOS, W.R.S. (Ed.) A produção animal na visão dos brasileiros. Piracicaba: Fundação de Estudos Agrários Luis de Queiroz, 2001. $927 p$.

CAPELLE, E.R.; VALADARES FILHO, S.C.; COELHO da SILVA, J.F, CECON, P. R.. Estimativas do valor energético a partir de características químicas e bromatológicas dos alimentos. Revista Brasileira de Zootecnia, v.6, p.1837-1856,

2001.http://dx.doi.org/10.1590/S151635982001000700022.

FARIA, V.P., MOURA, J.C., PEIXOTO, A.M.Cana-de-açúcar e seus subprodutos para bovinos In: SIMPÓSIO SOBRE NUTRIÇÃO DE BOVINOS, 5, 1993, Piracicaba. Anais... Piracicaba: FEALQ, 1993, p.176.

FARIA, V.P., MOURA, J.C., PEIXOTO, A.M. Nutrição de ruminantes - conceitos básicos e aplicados. 5.ed. Piracicaba: FEALQ, 1995. $563 p$.

FERNANDES, A.R.M.; SAMPAIO, A.A.M.; HENRIQUE, W.; PERECIN, D.; OLIVEIRA, E.A.; TÚLLIO, R.R. Avaliação econômica e desempenho de machos e fêmeas Canchim em confinamento alimentados com dietas à base de silagem de milho e concentrado ou cana-de-açúcar e concentrado contendo grãos de girassol. Revista Brasileira de Zootecnia, v.36, n.4, p.855-864, 2007.http://dx.doi.org/10.1590/S151635982007000400015

FIELD, R.A. Effect of castration on meat quality and quantity. Journal of Animal Science, v.32, n.5, p.849-856, 1971.

GESUALDI JÚNIOR, A.; PAULINO, M. F.; VALADARES FILHO, S.C.; SILVA, J.F.C.;
VELOSO, C.M.; CECON, P.R. Níveis de concentrado na dieta de novilhos F1 Limousin $x$ Nelore: consumo, conversão alimentar e ganho de peso.Revista Brasileira de Zootecnia, Viçosa- MG, v. 29 , n. 5 , p.1458-1466,

2000.http://dx.doi.org/10.1590/S151635982000000500026

ÍTAVO, L.C.V; DIAS, A.M.; ÍTAVO, C.C.B.F.; EUCLIDES FILHO, K.; MORAIS, M.G.; SILVA, F.F.; GOMES, R.C.; SILVA, J.P.B. Desempenho produtivo, características de carcaça e avaliação econômica de bovinos cruzados, castrados e não-castrados, terminados em pastagens de Brachiariadecumbens. Arquivo Brasileiro de Medicina Veterinária e Zootecnia,v.60, n.5, p.1157-1165, 2008.http://dx.doi.org/10.1590/S010209352008000500018.

MISSIO, R.L.; BRONDANI, I.L.; FREITAS, L.S.; SACHET, R.H.; SILVA, J.H.S.; RESTLE, J. Desempenho e avaliação econômica da terminação de tourinhos em confinamento alimentados com diferentes níveis de concentrado na dieta. Revista Brasileira de Zootecnia, v.38, n.7, p.1309-1316, 2009.http://dx.doi.org/10.1590/S151635982009000700021

NATIONAL RESEARCH COUNCIL NRC.Nutrient requirements of beef cattle. 7 rev. ed. National Academy Press, Washington, D.C.: 2000. 242p.

RESTLE, J.; VAZ, F.N.; FEIJÓ, G.L.D.; BRONDANI, I.L.; ALVES FILHO, D.C.; BERNARDES, R.A.C.; FATURI, C.; PACHECO, P.S. Características de carcaça de bovinos de corte inteiros ou castrados de diferentes composições raciais Charolês $\mathrm{x}$ Nelore. Revista Brasileira de Zootecnia, v. 29, n. 5, p. 1371-1379, 2000.http://dx.doi.org/10.1590/S151635982000000500015 SANTOS, E. M., ZANINE, A. M. Silagem de gramíneas tropicais. ColloquiumAgrariae, $v$. 2 n.1,p. $32-45$ 
http://dx.doi.org/10.5747/ca.2006.v02.n1.a2

1

SILVA, F.F. Aspectos produtivos da castração de novilhos de corte. Cadernos Técnicos de Veterinária eZootecnia, v.1, n.33, p.68-95, 2000.

ZANINE, A.M; SANTOS, E.M.; FERREIRA, D. J.; PEREIRA, O.G.. Populações microbianas e componentes nutricionais nos órgãos do capim-tanzânia antes e após a ensilagem. Semina - Ciências Agrárias, v.28, n.1, p.143150, 2007.http://dx.doi.org/10.5433/16790359.2007v28n1p143 